\title{
Molecular Characterization of the Highest Risk Adult Patients with Acute Myeloid Leukemia (AML) through Multi-omics Clustering
}

Trinh Nguyen ( $\nabla$ tinh.nguyen@nih.gov )

National Cancer Institute

John W Pepper

National Cancer Institute

Cu Nguyen

National Cancer Institute

Yu Fan

National Cancer Institute

Ying Hu

National Cancer Institute

Qingrong Chen

National Cancer Institute

Chunhua Yan

National Cancer Institute

Daoud Meerzaman

National Cancer Institute

\section{Research Article}

Keywords: multi-omics, unsupervised clustering, intrinsic subtypes, acute myeloid leukemia

Posted Date: July 8th, 2021

DOI: https://doi.org/10.21203/rs.3.rs-138491/v2

License: (1) (1) This work is licensed under a Creative Commons Attribution 4.0 International License.

Read Full License 


\title{
Molecular characterization of the highest risk adult patients with acute
}

\section{myeloid leukemia (AML) through multi-omics clustering}

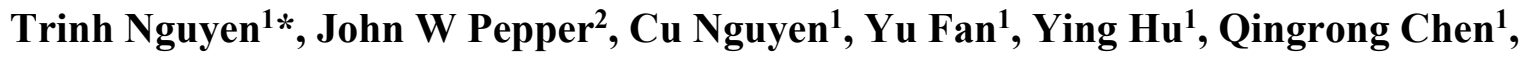 Chunhua Yan', Daoud Meerzaman'}

${ }^{1}$ Center for Biomedical Informatics and Information Technology, National Cancer Institute, Rockville, Maryland, United States of America

${ }^{2}$ Division of Cancer Prevention, National Cancer Institute, Rockville, Maryland, United States of America

*Corresponding author: tinh.nguyen@nih.gov

\begin{abstract}
Background: Acute myeloid leukemia (AML) is a clinically heterogeneous group of diseases with poor outcomes that are partly due to its complex and poorly understood heterogeneity. Methods: Here, we use a multi-omics approach to identify a molecular subgroup with the worst response to chemotherapy, and to identify promising drug targets specifically for this AML subgroup.
\end{abstract}

Results: Multi-omics clustering analysis using RNA and CNA expression data resulted in the three primary clusters of 166 AML adult TCGA cancer cases. One of these clusters, which we label as the high-risk molecular subgroup (HRMS), consisted of cases that responded very poorly to standard chemotherapy, with only about 10\% survival to two years. The gene TP53 was mutated in most cases in the HRMS, but not in other cases. The top 6 genes over-expressed in the HRMS included E2F4, CD34, CD109, MN1, MMLT3, and CD200. Multi-omics pathway analysis using RNA and CNA expression data identified in the HRMS subgroup over-expressed pathways related to immune function, cell proliferation, and DNA damage.

Conclusion: Some AML patients are not successfully treated with the current standard of care chemotherapy, and urgently need targeted therapeutics. Potential drug targets include overexpressed genes $E 2 F 4$, and $M N 1$, as well as mutations in TP53, and several molecular pathways.

Keywords: multi-omics, unsupervised clustering, intrinsic subtypes, acute myeloid leukemia. 


\section{Introduction}

AML not only represents one of the most fatal leukemias but also ranks among the deadliest of all cancers. It presents a myriad of chromosomal alterations and gene mutations, comprising a clinically heterogeneous group of diseases [1]. The poor outcomes of AML are at least in part due to its complex and poorly understood heterogeneity, which has impeded the rational targeting of defined molecular subgroups [1]. The most widely used criteria for clinical subgroups are based on cytogenetic abnormalities, which do not translate directly or easily into drug targets. Although over- and under-expression of genes is known to affect prognosis, such data are not included in the most recent revision of the WHO classification of subgroups [2]. AML treatment concepts have not drastically changed since the 1970s, and AML generally remains a mostly incurable disease [1]. For many other cancers, effective targeted therapeutics have become available, but treatment of AML continues to rely mostly on untargeted therapies. This is largely due to the difficulties of molecular targeting for an extremely heterogeneous collection of diseases.

The current standard of care for AML, using untargeted chemotherapy, is effective against those cytogenetic subgroups recognized as having good prognosis under the current treatment regimen, but chemotherapy offers very low survival rates to those cytogenetic subgroups recognized as having poor prognosis under this treatment regimen, with only about $20 \%$ survival beyond two years [3]. To address this problem, we examined multi-omics data to seek intrinsic molecular subgroups that could potentially guide the development of more effective targeted therapies specifically for those patients who would respond poorly to untargeted chemotherapy. The existing system of AML subtyping predates many modern techniques for molecular analysis. 


\section{Methods}

We began with an unsupervised clustering analysis using two types of data: somatic copy number alteration (CNA), and gene expression levels from RNA-seq measurements. We then identified differences among the three resulting clusters in their risk stratification, and in overall survival, using datasets with information on mutations, putative copy number alterations from GISTIC (Genomic Identification of Significant Targets in Cancer), with matched clinical data. Next, we performed pathway analyses to find differences in which molecular pathways were enriched in the three molecular subgroups we found. Further analyses focused on molecular characterization of the one cluster with the worst outcomes under the current treatment regimen.

\section{Dataset preparation}

We downloaded the TCGA adult AML datasets directly from https://www.cbioportal.org/study/clinicalData?id=laml_tcga_pub. We used the total of 166 samples with transcriptomic, copy number alteration, mutation, and clinical datasets. These samples were obtained from peripheral blood and represented the major morphologic and cytogenetic subgroups of AML [3]. We used two different CNA datasets: CNA segmentation and discrete CNA values datasets.

\section{Calculation of CNA segment}

We estimated gene level CNA as the segment mean of copy numbers of the genomic region of a gene by using TCGA-Assembler 2 [4] downloaded from https://github.com/compgenome365/TCGA-Assembler-2 (version 2.0.6). Degree of CNA was calculated as $\log 2$ (tumor values/normal values). Across samples, CNA of all genes had a standard deviation greater than the median. Therefore, in order to exclude near normal (very low) CNA values, only genes with a sum of CNA values across samples greater than zero were used for analysis, resulting in 13,019 genes total. $\mathrm{Hg} 19$ annotation was used to obtain gene position. 


\section{Discrete CNA values}

Putative copy-number calls determined using GISTIC 2.0 were used to obtain the information for six genes known to be important for AML: FLT3, NPM1, DNMT3A, CEBPA, RUNX1, and RUNX1T1. Patients with CNA values greater than or equal to 1 were classified as copy number amplifications, while patients with values less than or equal to -1 were classified as copy number deletions. Patients with zero values were classified as unchanged.

\section{RNA-seq expression}

We used RSEM (RNA-Seq by Expectation Maximization) expected raw count expression dataset. Genes without at least 1 count-per-million reads in at least $50 \%$ of the total samples were filtered out. The resulting RNA dataset was $\log 2$ transformed and quantile normalized. A total of 12,934 genes were retained for analysis.

\section{Mutation dataset}

The AML genes RUNX1, RUNX1T1, CEBPA, FLT3, NPM1, DNMT3A, and TP53 were analyzed across 166 samples.

\section{Clinical dataset}

The clinical dataset provided information such as cytogenetic abnormalities and the risk stratification.

\section{Pathway database}

We downloaded the gmt file of MSigDB hallmark gene set collection (version 7.1) from https://www.gsea-msigdb.org/gsea/msigdb/collections.jsp for annotation. The 50 hallmarks in this collection represent biological state or process [5].

\section{Multiple omics data integrative clustering and gene set analysis (MOGSA)}

MOGSA is an R software package for multivariate single sample gene set analysis [6]. Using this package (version 1.22.1), we integrated transcriptomic data and gene level CNA over the same set of samples. Based on the chosen PCs from multiple factorial analysis (MFA), we 
performed unsupervised clustering to identify subgroups and calculated Hall Mark gene set pathway scores for individual samples.

\section{Determining the number of principle components}

MFA [7] was incorporated into the moa function from MOGSA to perform principal component analysis (PCA) for CNA and RNA-seq Expression. The first 6 PCs were chosen for downstream analysis due to the equal contribution of CAN and RNA-seq expression (figure 1)

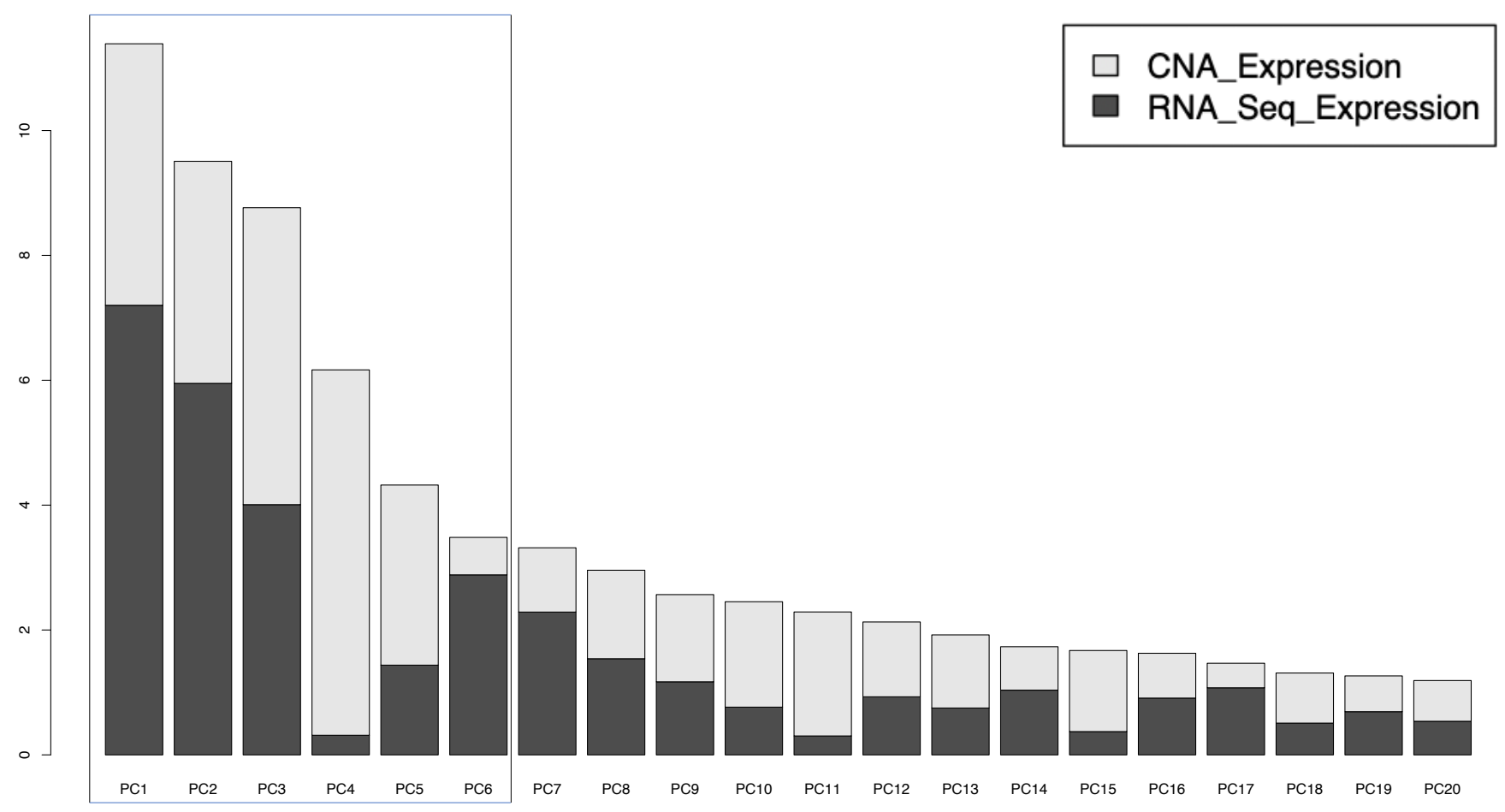

Figure 1. Distribution of variances explained by the 20 principal components (PCs). The first six PCs were used to identify subgroups by clustering. These contained a total of $43.6 \%$ of total variance, with CNA and Gene expression contributing equally.

\section{Identifying subgroups through unsupervised clustering}

In order to recognize natural subgroups among the cases, we used ConsensusClusterPlus (version 1.52.0) [8] to identify clusters based on the first six PCs (Figure 1). We used these parameter settings: $\operatorname{maxK}=6$, reps $=10000$, pItem=0.8, clusterAlg="hc", finalLinkage= "ward.D2", 
distance="pearson". As the best clustering solution, we chose 3 clusters because this number of clusters gave the greatest area under the CDF curve (figure 2A), and the best separation of clusters (figures $2 \mathrm{~B}$ and $2 \mathrm{C}$ ). We named the three resulting clusters as follows: $\mathrm{C} 1$ or 'Intermediate Risk Molecular Subgroup'; C2 or 'Low Risk Molecular Subgroup'; and C3 or 'High Risk Molecular Subgroup'. Descriptive names were based on our survival analysis (Figure 3A).

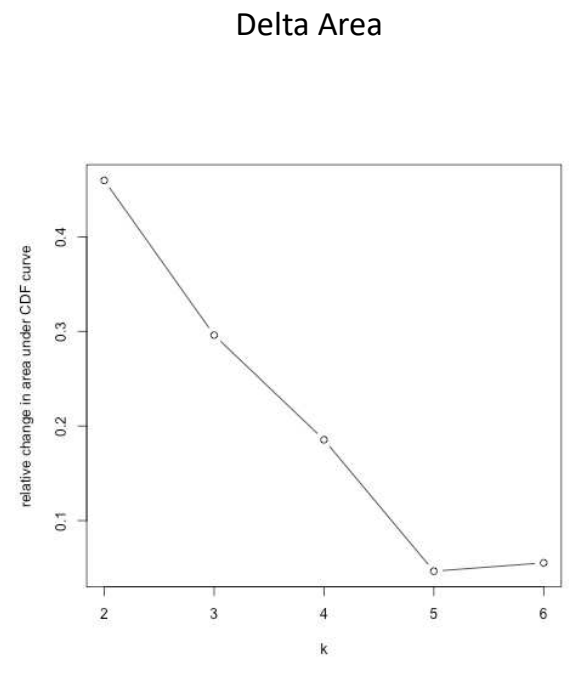

Figure $2 \mathrm{~A}$

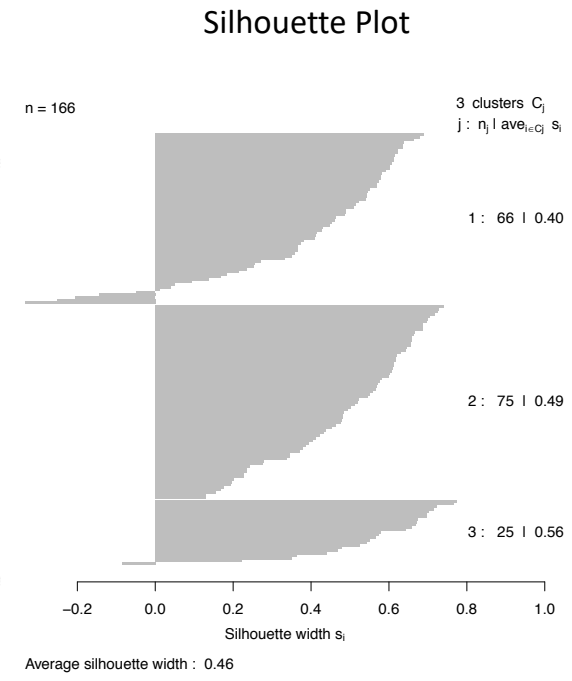

Figure 2 B

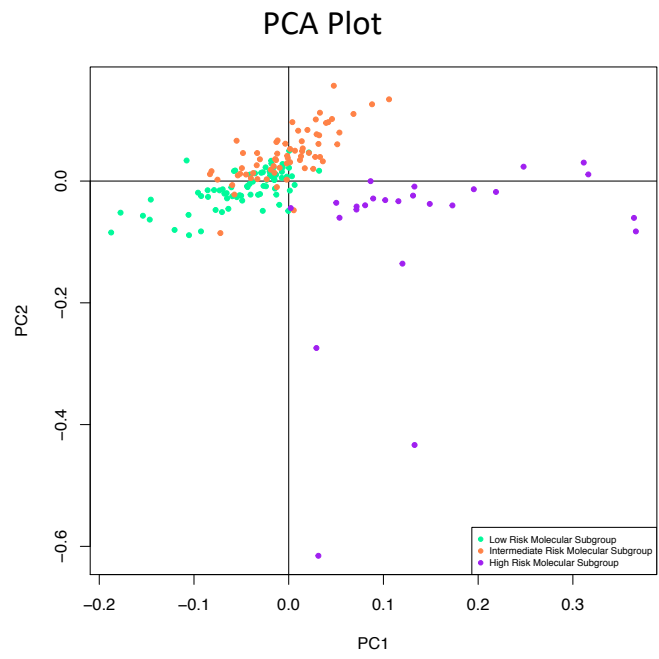

Figure $2 \mathrm{C}$

Figure 2. (A) Delta area shows the numbers of clusters (k) (X axis) and their relative change in area under CDF curve (Y-axis). (B) Silhouette plot of chosen clusters with k=3. (C) The separation of 3 subgroups: Low Risk Molecular Subgroup, Intermediate Risk Molecular Subgroup, and High Risk Molecular Subgroup.

\section{Survival analysis}

We used the R modules Survfit and coxph [9] to perform overall survival analysis based on the three subgroups resulting from the total of 166 TCGA adult samples .. 


\section{Calculating gene-set pathway scores}

We used the MOGSA (the Integrative Single Sample Gene-set Analysis of Multiple Omics Data) package, to identify the MSigDB hallmark pathways' gene set scores (GSS). We used these parameter settings: $\mathrm{nf}=6$, proc.row="center_ssq1", w.data= "lambda 1", and statis=FALSE.

\section{Selecting representative molecular pathways for three subtypes}

In order to choose representative molecular pathways, we firstly selected the pathways resulting from the MOGSA function with GSS FDR (false discovery rate) values smaller than 0.01 in 50\% of all samples. Secondly, we used the R functions, fitting generalized linear models (GLM) to calculate the difference of GSSs in each subgroup vs. that in the rest and selected the top 5 and bottom 5 representative pathways ranked by GLM T values, resulting in 16 unique representative pathways total with GLM FDR $<0.01$. The 3 subgroups were also different significantly in these representative pathways with ANOVA test FDR $<0.001$. Lastly, we visualized z-score scaled median GSSs in a heatmap to show the overall enrichment from both datatypes as well as the contribution of each datatype to the subgroups (Figure 3B).

\section{Cytogenetics information of the total of 166 patients}

The patients' information on cytogenetic risk and their genetic abnormalities is shown in Supplemental Table 1. 


\section{Results}

The three putative subgroups of cases resulting from our clustering analysis (Figure 2) did appear to differ meaninfully from each other. They differed both in their prognosis (Figure 3A), and their molecular traits (Figure 3B).
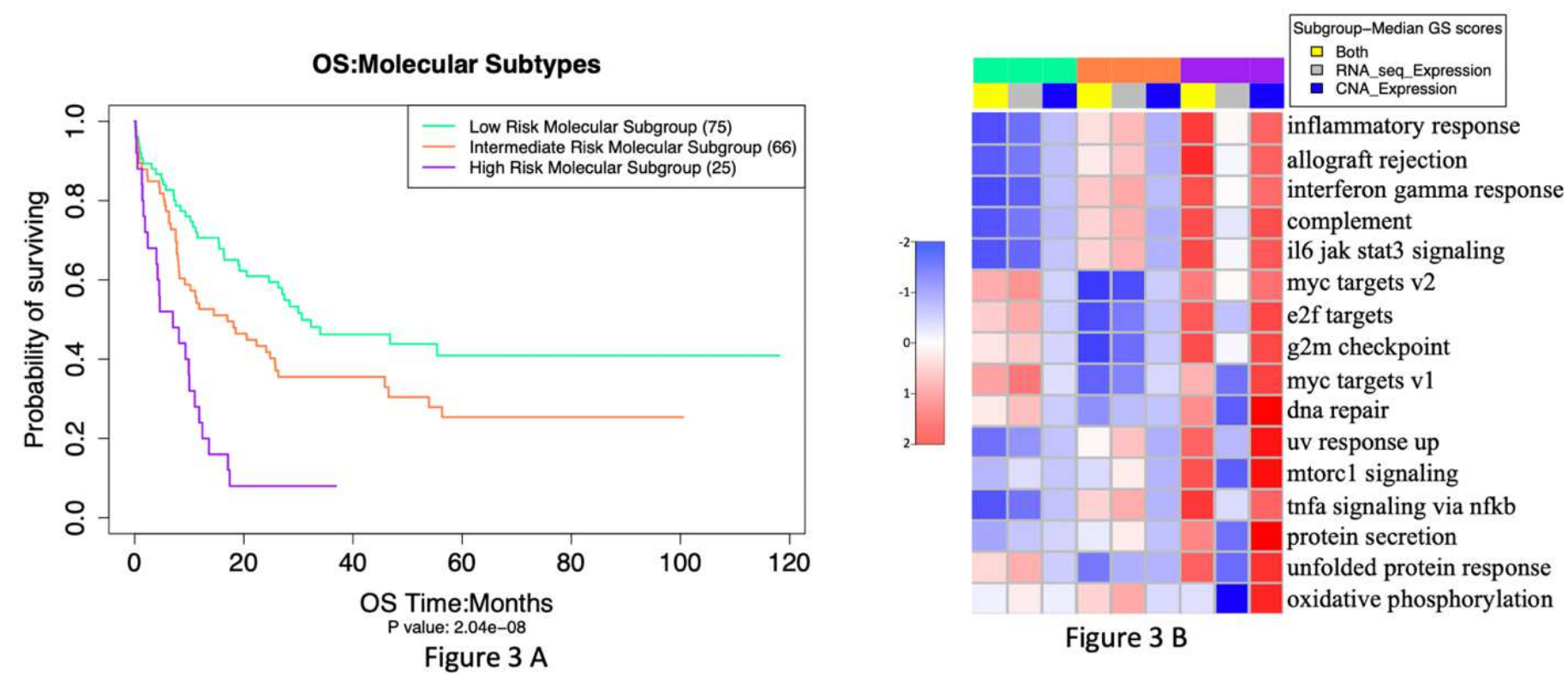

Figure 3 B

Figure 3. (A): Overall Survival outcomes of our three molecular subgroups differd significantly ( $\mathrm{p}=2 \times 10^{-8}$ (adjusted by gender and age); (B): Multi-omic expression analysis of molecular subgroups. 
These three putative AML subgroups also differed in several aspects of their molecular makeup (Figure 4).

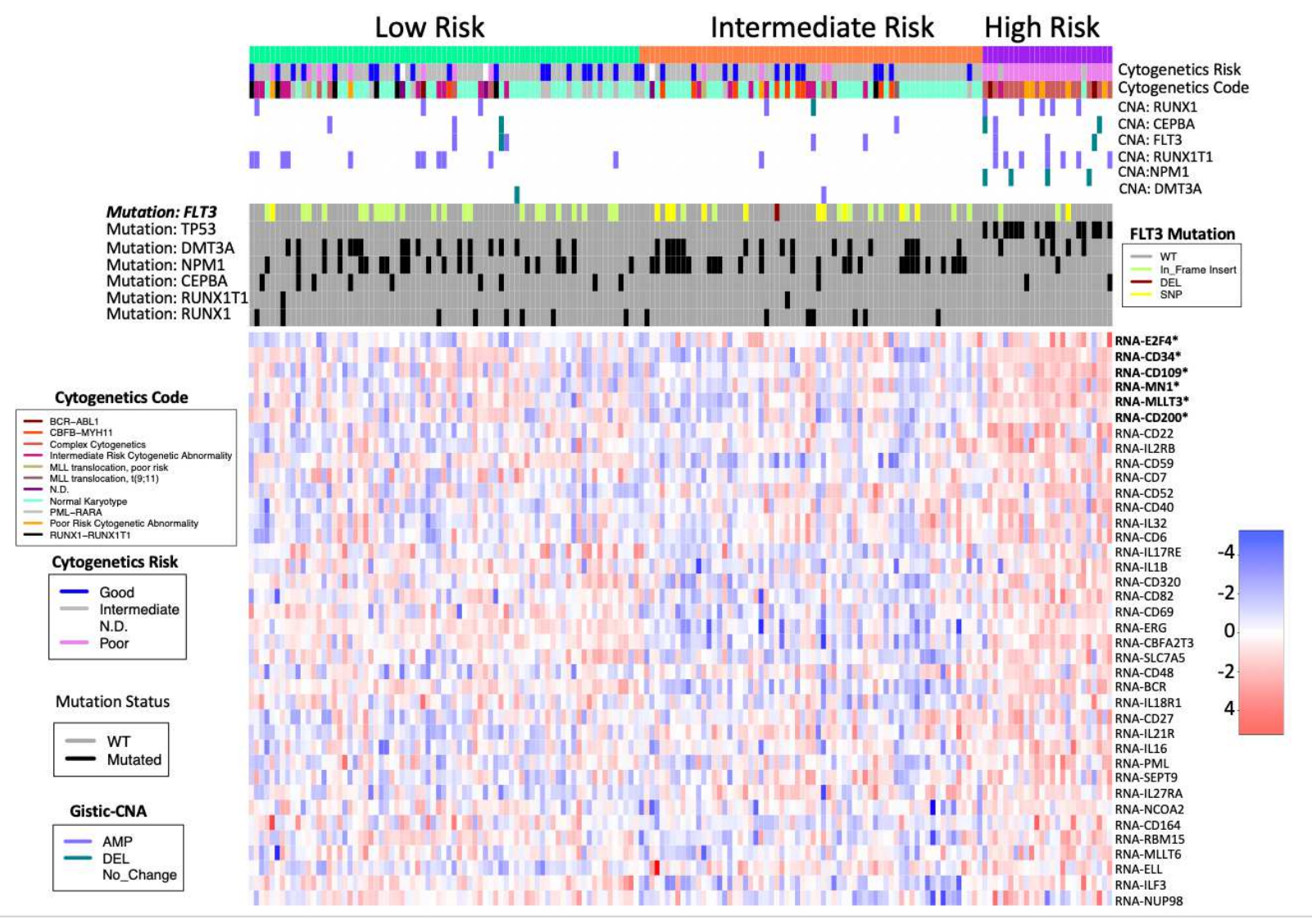

Figure 4. Summary of molecular differences among the three patient subgroups. The six genes with the most elevated expression in our HRMS are marked with * in the list of gene names.

Among the six AML genes we examined, the HRMS had significantly fewer gene mutations than the other patients. In contrast, among these six AML genes, the HRMS had a higher frequency than the other patients of copy number alterations (CNAs) (Figure 4). When separated by CNA type, this difference was statistically significant for copy-number amplifications using Fisher exact test $(p=0.03)$, but not for copy-number deletions $(p=0.08)$.

The patients in our HRMS had significantly lower overall survival than did other patients (Figure 3A). This was largely consistent with their risk stratification based on cytogenetics (Table 1). 
The three new molecular subgroups were significantly associated with established cytogenetic risk stratifications from clinical data (Table 1) with $\mathrm{P}$ value $<10^{-14}$ by Fisher's exact test. Among our samples, most patients with a 'poor' cytogenetic risk classification fell within our multi-omics HRMS, while all those with a 'good' cytogenetic risk classification fell into other subgroups. As expected, based on this association with established poor cytogenetic risk stratification, HRMS patients had poor overall survival. However, HRMS included only a subset of the poor cytogenetic risk group patients in our dataset (23 of 35 total, see Table 1), and this subset had even worse survival than did cytogenetic poor risk patients as a whole.

Table 1. Counts of patients in our three molecular subgroups, as classified by established cytogenic risk levels. Details of the cytogenetic abnormalities associated with each cytogenetic risk category are provided in Supplemental Table 1.

\begin{tabular}{|l|l|l|l|}
\hline $\begin{array}{l}\text { Cytogenic Risk } \\
\text { Stratification }\end{array}$ & $\begin{array}{l}\text { Low Risk } \\
\text { Molecular Subgroup }\end{array}$ & $\begin{array}{l}\text { Intermediate Risk Molecular } \\
\text { Subgroup }\end{array}$ & $\begin{array}{l}\text { High Risk Molecular } \\
\text { Subgroup }\end{array}$ \\
\hline Good & 19 & 13 & 0 \\
\hline Intermediate & 46 & 48 & 2 \\
\hline Not determined & 2 & 1 & 0 \\
\hline Poor & 8 & 4 & 23 \\
\hline
\end{tabular}

In a previous analysis, overall survival of patients in the poor cytogenetic risk group at two years was reportedly about 20\% [3] (we replicated this survival analysis with our subset of 166 of the 200 patients used in the earlier study, see Supplemental Figure 1). In contrast, among the patients in our HRMS, overall survival at two years was worse, at only about $10 \%$ (Figure 3A).

Because patients in our HRMS had significantly worse clinical outcomes than other patients, we focused on this molecular subgroup for further molecular characterization.

The multi-omics pathway analysis using RNA and CNA expression data revealed significant differences among the molecular subgroups in the combined activation GS scores of various molecular pathways from both datasets (see Supplemental Table 2 for the GSS of these pathways). The HRMS showed higher activation than other patients of most molecular pathways 
related to immune function, cell proliferation, and DNA damage where CNA expression contribute more than RNA-seq expression to this overall GS (Figure 3B).

\section{Gene mutations}

Among genes known to be important in AML, mutation frequencies differed in our HRMS versus other patients (Table 2). Among the seven AML genes in our mutation data set, most (4/7) had lower mutation frequencies in HRMS than in other patients, but these differences were not statistically significant. In contrast to the other AML genes, TP53 was mutated in most patients in the HRMS, but not in any other patients, constituting a highly significant difference (Table 2).

Table 2. Frequencies of mutation in AML genes in HRMS, versus other patients. P-values are from Fisher's exact tests on counts of mutant and wild-type genes.

\begin{tabular}{|l|l|l|l|}
\hline Gene & High Risk frequency & Non-High Risk frequency & $\begin{array}{l}\mathrm{p} \text { value } \\
(<0.01)\end{array}$ \\
\hline RUNX1 & $0 \%$ & $11 \%$ & NS \\
\hline RUNXIT1 & 0 & $1.4 \%$ & NS \\
\hline CEBPA & $8 \%$ & $7.1 \%$ & NS \\
\hline FLT3 & $12 \%$ & $31 \%$ & NS \\
\hline NPMI & $4 \%$ & $33 \%$ & $0.003^{* *}$ \\
\hline DNMT3A & $20 \%$ & $24 \%$ & NS \\
\hline TP53 & $56 \%$ & 0 & $10^{-10}$ \\
\hline
\end{tabular}

Note: NS: nonsignificant, and **: significant

\section{Gene over-expression}

The dataset for RNA-seq included seven prominent genes known to be important in AML: FLT3, DMT3A, NPM1, CEPBA, RUNX1, E2F4, and TP53.

Six of the seven genes listed above, excluding $C E P B A$, varied significantly among clusters (ANOVA test, $\mathrm{p}<0.01$ ). Only one of these genes, E2F4 had elevated expression in the HRMS subgroup (Figure 5). This difference was highly significant $\left(\mathrm{p}<10^{-5}\right)$. 


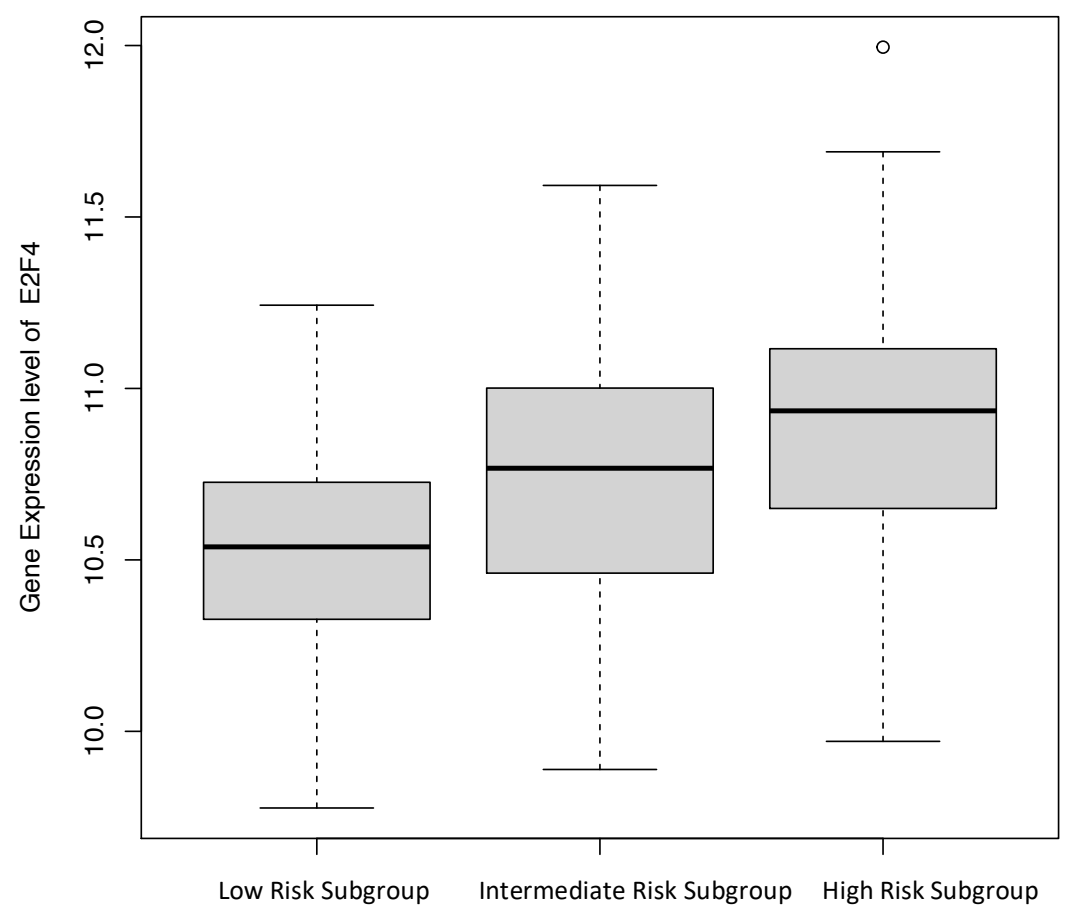

Figure 5: E2F4 gene expression was elevated in the High Risk Molecular Subgroup with $\mathrm{P}<10^{-5}$

In addition to the seven genes listed above, we compiled a total of 139 AML genes expression involved in rearrangement, immune interaction, and blast from Mitelman database, OMIM, and publications (see Supplemental Table 3, "Known AML genes").

From this list, we identified thirty-seven genes that were the most over-expressed in the high-risk HRMS subgroup relative to other cases. Each of these genes differed significantly among clusters (ANOVA test, $\mathrm{p}<0.01$ ). Among these genes, the top five genes for this difference, ranked by magnitude of over-expression, were CD34,CD109, MN1, MLLT3, and CD200 (Figure 4). 


\section{Discussion}

The high-risk HMRS subgroup that we identify here, based on molecular markers, and overlaps substantially with the long-established cytogenetic high-risk subtype (see Table 1), but differs in two important ways. Firstly, after treatment with chemotherapy, The HMRS subgroup had even lower survival -than does the cytogenetic high-risk subtype. Thus, our molecular subgroup offers a more focused classification of cases that are not successfully treated with the current standardof-care chemotherapy, and that therefore urgently need targeted therapeutics. Secondly, unlike cytogenetic features, which do not offer drug targets, this subgroup is characterized by molecular traits that do offer potential as new drug targets. Our findings indicate several candidates for drug targets specific to the extremely high-risk patients of our HRMS. These candidate targets include mutations of gene TP53, which was mutated in most HRMS patients (Table 2), as well as overexpression of six genes that were highly over-expressed in the HRMS, including CD34, CD109, CD200, E2F4, MN1, and MLLT3. Other potential targets may be found in the molecular pathways that are highly activated in our HRMS subgroup.

One of the strongest molecular associations with our HRMS subgroup is mutation in TP53. It has long been established that TP53 mutations are associated with resistance to chemotherapy and with short survival in hematologic malignancies [10]. The importance of TP53 mutations specifically for our HRMS subgroup is also consistent with the guidelines of the National Comprehensive Cancer Network, which classify AML patients with normal cytogenetics into the poor/adverse risk category if they harbor TP53 mutations [11]. In AML, mutations in TP53 are associated with poor responses to chemotherapy, and with very poor prognosis [12]. These authors [12] suggested that it was important to test whether other pathways activated by TP53 mutations could be therapeutically targeted. Our results should contribute to reaching that goal.

Overexpression of MN1 is known to confer resistance to chemotherapy, and a worse AML prognosis. Pardee [13] investigated the mechanisms for this and suggested that therapies directed at increasing TP53 function may be useful for such patients.

Another of the genes most over-expressed in our HRMS subgroup was E2F4. This is unsurprising, as it is known that TP53 mutations can drive the expression of E2F4 [14]. The over-expression of E2F4 in our HRMS subgroup was also consistent with a recent report that $\mathrm{E} 2 \mathrm{~F} 4$ over-expression was associated with poor prognosis in AML patients, and that in a mouse 
model, depleting E2F4 inhibited proliferation and suppressed the growth of AML cells [15]. These authors suggested E2F4 as a potential therapeutic target [15], and here we support that suggestion by showing the importance of this gene specifically in the HRMS subgroup of patients expected to fare worst under untargeted chemotherapy.

Other molecular characteristics of our HRMS include highly activated molecular pathways in the categories of immune function, DNA damage, and cell proliferation, all three of which are consistent with previous reports. A high level of DNA damage has been reported for cells of AML patients categorized as having high-risk cytogenetics and is accompanied by activation of DNA damage pathway [16]. Our results show that inflammatory response and IL6 JAK STAT signaling pathways were highly activated in HRMS. This is consistent with the findings that the inflammatory pathway leads to an activation of the JAK/STAT signaling in AML which fosters leukemia proliferation [17].

Our results suggest that pathways activated by mutations in TP53 might be targeted therapeutically. We found that the pathways highly activated in our HRMS are in the proliferation category, including, E2F targets, G2M checkpoint, and Myc targets V2 (Supplemental Figure 2). Activation of these proliferation pathways can be promoted by the overexpression of the E2F4 gene.

\section{Conclusions}

An identifiable subset of AML patients is not successfully treated with the current standard of care chemotherapy, and urgently need targeted therapeutics. Potential drug targets include overexpressed genes E2F4, and MN1, as well as mutations in TP53, and several molecular pathways.

In this HRMS, we have identified several over-expressed genes including E2F4. In addition, the presence of TP53 mutations in most of the samples in this subgroup probably contributed to poor responses to chemotherapy, and to poor prognosis. TP53 mutations may drive the elevated transcriptomic expression of E2F4. We have found that E2F targets, G2M checkpoint, and Myc targets V2 are highly activated in this HRMS. We suggest that some potential therapeutic targets include over-expressed genes E2F4, and MN1, as well as mutations in TP53 for this specific HRMS. 
Abbreviations:

AML: acute myeloid leukemia; TCGA: the cancer genome atlas; CNA: copy number alteration; RNA-Seq: transcriptomics sequencing; RSEM: RNA-Seq by Expectation Maximization; MOGSA: multiple omics data integrative clustering and gene set analysis; MFA: multiple factorial analysis; PCA: principal component analysis; GSS: gene set scores; FDR: false discovery rate; HRMS: High Risk Molecular Subgroup.

Ethics approval and consent to participate:

Not applicable

Consent for publication:

Not applicable.

Competing interests:

The authors have no competing interests to report.

Funding:

There is no funding.

Availability of data and material:

All data used in this study can be accessed from https://www.cbioportal.org/study/clinicalData?id=laml tcga pub.

Codes used in this manuscript are available upon request.

Authors' contributions:

T.N planned, carried out the analysis and wrote the manuscript. C.N helped with the input data. J.P., C.N., Y.F., C.Y., Q.R., D.M., provided advice for carrying out the analysis and for the manuscript. All authors reviewed the manuscript.

Acknowledgements:

Not applicable. 


\section{$\underline{\text { References }}$}

1. Green SD, Konig H. Treatment of Acute Myeloid Leukemia in the Era of GenomicsAchievements and Persisting Challenges. Frontiers in Genetics 2020;11

2. Vardiman JW, Thiele J, Arber DA, Brunning RD, Borowitz MJ, Porwit A, et al. The 2008 revision of the World Health Organization (WHO) classification of myeloid neoplasms and acute leukemia: rationale and important changes. Blood 2009;114:937-51

3. Ley TJ. Genomic and Epigenomic Landscapes of Adult De Novo Acute Myeloid Leukemia (vol 368, pg 2059, 2013). New England Journal of Medicine 2013;369:98-

4. Wei L, Jin ZL, Yang SJ, Xu YX, Zhu YT, Ji Y. TCGA-assembler 2: software pipeline for retrieval and processing of TCGA/CPTAC data. Bioinformatics 2018;34:1615-7

5. Liberzon A, Birger C, Thorvaldsdottir H, Ghandi M, Mesirov JP, Tamayo P. The Molecular Signatures Database Hallmark Gene Set Collection. Cell Systems 2015;1:41725

6. Meng C, Basunia A, Peters B, Gholami AM, Kuster B, Culhane AC. MOGSA: Integrative Single Sample Gene-set Analysis of Multiple Omics Data. Molecular \& Cellular Proteomics 2019;18:S153-S68

7. Abdi H, Williams LJ, Valentin D. Multiple factor analysis: principal component analysis for multitable and multiblock data sets. Wiley Interdisciplinary Reviews-Computational Statistics 2013;5:149-79

8. Wilkerson MD, Hayes DN. ConsensusClusterPlus: a class discovery tool with confidence assessments and item tracking. Bioinformatics 2010;26:1572-3

9. Li JCA. Modeling survival data: Extending the Cox model. Sociological Methods \& Research 2003;32:117-20 
10. Wattel E, Preudhomme C, Hecquet B, Vanrumbeke M, Quesnel B, Dervite I, et al. P53 MUTATIONS ARE ASSOCIATED WITH RESISTANCE TO CHEMOTHERAPY AND SHORT SURVIVAL IN HEMATOLOGIC MALIGNANCIES. Blood $1994 ; 84: 3148-57$

11. Daver N, Schlenk RF, Russell NH, Levis MJ. Targeting FLT3 mutations in AML: review of current knowledge and evidence. Leukemia 2019;33:299-312

12. Wang Y, Liu Y, Bailey C, Zhang HX, He M, Sun DX, et al. Therapeutic targeting of TP53-mutated acute myeloid leukemia by inhibiting HIF-1 alpha with echinomycin. Oncogene 2020;39:3015-27

13. Pardee TS. Overexpression of MN1 Confers Resistance to Chemotherapy, Accelerates Leukemia Onset, and Suppresses p53 and Bim Induction. Plos One 2012;7

14. Blandino G, Di Agostino S. New therapeutic strategies to treat human cancers expressing mutant p53 proteins. Journal of Experimental \& Clinical Cancer Research 2018;37

15. Feng YB, Li LL, Du Y, Peng XQ, Chen FH. E2F4 functions as a tumour suppressor in acute myeloid leukaemia via inhibition of the MAPK signalling pathway by binding to EZH2. Journal of Cellular and Molecular Medicine 2020;24:2157-68

16. Cavelier C, Didier C, Prade N, Mas VMD, Manenti S, Recher C, et al. Constitutive Activation of the DNA Damage Signaling Pathway in Acute Myeloid Leukemia with Complex Karyotype: Potential Importance for Checkpoint Targeting Therapy. Cancer Research 2009;69:8652-61

17. Habbel J, Arnold L, Chen YY, Mollmann M, Bruderek K, Brandau S, et al. Inflammation-driven activation of JAK/STAT signaling reversibly accelerates acute myeloid leukemia in vitro. Blood Advances 2020;4:3000-10 
Additional files:

1.

File name: Sup_Figure_1

Title of data: Overall Survival Cytogenetics Risks Groups

Description of data: Supplemental Figure 1. Overall Survival analysis based on cytogenetics risk stratification ( $\mathrm{P}$ val. adjusted by gender and age: 9 x 10-9).

2.

File name: Sup_Figure_2

Title of data: N.A

Description of data: Comparisons among the three novel multi-omics subgroups in gene activation scores for six molecular pathways

3.

File name: Sup_Table_1.pdf

Title of data: N.A

Description of data: Cytogenetic risk stratification from clinical dataset 4.

File name: Sup_table_2_Of_GSS_RepresentativePathways.csv

Title of data: GSS Representative Pathways

Description of data: single sample Gene Set Scores of 16 representative pathways.

5.

File name: Sup_table_3.txt

Title of data: Known AML genes

Description of data: Important AML genes from Mitelman database, OMIM, and publications. 


\section{Supplementary Files}

This is a list of supplementary files associated with this preprint. Click to download.

- SupFigure1.pdf

- SupFigure2.pdf

- SupTable1.pdf

- Suptable2OfGSSRepresentativePathways.csv

- Suptable3.txt 